\title{
World Cancer Day 2021: Remembering the ongoing cancer pandemic
}

\author{
William Ying Khee Hwang, ${ }^{1,2,3}$ FAMS, Tobias Khoo, ${ }^{4}$
}

The COVID-19 pandemic has taken the world by storm, affecting millions of lives, plundering multiple economies and dramatically changing our way of life. By 8 March 2021, the World Health Organization had already reported 116,521,281 confirmed cases of COVID-19 around the world, including 2,589,548 deaths, giving a case fatality rate of $2 \%$. In Singapore, there were 60,033 confirmed cases of COVID-19 with 29 deaths at that time, with a case fatality rate of $0.05 \%$.

During the same period, another ongoing pandemic continued to rage around the world, affecting 18,094,716 people and claiming 9,894,402 lives-far more deaths than COVID-19. ${ }^{2}$ This deadly blight on global health is the cancer pandemic, which is the second leading cause of death around the world and the top cause of death in Singapore, where the incidence of malignancies has increased nearly six times in the last 50 years., ${ }^{3,4}$ The ageing population around the world has contributed to the rising number of new cancer cases globally. ${ }^{5}$ Cancer also strikes vulnerable populations, with the most significant increases in mortality occurring in low- and middleincome countries, which account for over 5 million deaths due to cancer every year. ${ }^{6}$

The COVID-19 pandemic has significantly affected oncology patients around the world through delays in cancer screening, impacts on the delivery of cancer care, and adverse outcomes in patients with cancer who develop COVID-19, as well as causing severe disruption to cancer research. ${ }^{7}$ Patient enrolment in cancer clinical trials has been severely affected and there are also risks of missing or delayed data collection from ongoing trials. ${ }^{8}$

There is a need, therefore, to continue support for cancer control because cancer is a far more protracted illness with a high incidence of relapses and long-term complications despite many new treatments in recent years. Lack of accessible cancer screening during the COVID-19 pandemic in many countries has resulted in delay in cancer detection. In the UK alone, approximately 1 million people were not invited for colorectal cancer screening when they were due, which results in a risk of patients presenting with late-stage cancer when restrictions are lifted. It is feared that this could lead to a spike in cancer mortality in the coming year as previous research has shown that just a 4-week delay in treatment could be associated with an increase in mortality rate across all common forms of cancer treatment, with longer delays being increasingly deadly. ${ }^{9}$ Gains in survival might be achieved if we prioritise more effort into minimising the time from cancer screening and diagnosis to treatment. Continued support for oncology research is also vital to ensure long-term control of the cancer pandemic.

When COVID-19 hit Singapore, the government implemented measures to prevent its spread. One major measure was the circuit breaker-Singapore's version of lockdown - from 7 April 2020 to 1 June 2020. During the circuit breaker period, cancer screening and surveillance services, like the national mammographic screening programme, were categorised as non-essential and had to be temporarily halted during the circuit breaker. Outpatient visits were also minimised as much as possible and were replaced with teleconsultations as well as home medication delivery. ${ }^{10,11}$ This resulted in a $30 \%$ decrease in cancer consultations, which may have led to some potential delayed diagnoses. However, maximal efforts were taken to minimise the impact on our patients while delivering optimal cancer care. Fortunately, because community spread was kept under control, cancer services have been able to resume to some extent. However, beyond short-term measures, it is important to restructure the screening, consultation and treatment of patients to ensure that they will continue to receive the best preventive and therapeutic care in the long term. Meanwhile, the measures that have so far been instituted are likely to improve the quality of cancer care for our patients by reducing their time in spent in the cancer centre as well as their need to travel to the centre. ${ }^{10}$

The speed with which scientists have come up with quick and reliable test kits for COVID-19 is remarkable. Even more amazing are the multiple vaccines produced in record time using cutting-edge technology. If new oncology test kits (e.g. liquid biopsies) could be employed

\footnotetext{
${ }^{1}$ Department of Haematology, Singapore General Hospital, Singapore

${ }^{2}$ Division of Medical Oncology, National Cancer Centre Singapore, Singapore

${ }^{3}$ Duke-NUS Medical School, Singapore

${ }^{4}$ Raffles Junior College, Singapore

Correspondence: Prof William YK Hwang, Executive Offices, National Cancer Centre Singapore, 11 Hospital Crescent, Singapore 169610.

Email: william.hwang.y.k@singhealth.com.sg
} 
with the speed and accuracy of COVID-19 testing in future, this could open significant possibilities for cancer screening, diagnosis and follow-up. Also, if mRNA and other vaccines could be rolled out for use in oncology as quickly as they have been for COVID-19, this could revolutionise the field on immunotherapeutics for cancer. If such a degree of global cooperation, scientific ingenuity as well as pooling of data and resources was employed in oncology, then we could actually stand a chance of finally taming the cancer pandemic.

\section{REFERENCES}

1. World Health Organization.WHO Coronavirus (COVID-19) Dashboard. Available at: https://covid19.who.int/. Accessed on 8 March 2021

2. World Health Organization, International Agency for Research on Cancer. Cancer Today, December 2020. Available at: https://gco.iarc. $\mathrm{fr} /$ today/data/factsheets/cancers/40-All-cancers-excluding-nonmelanoma-skin-cancer-fact-sheet.pdf. Accessed on 14 Jan 2021.

3. Ministry of Health Singapore. Principal Causes of Death. Available at: https://www.moh.gov.sg/resources-statistics/singapore-healthfacts/principal-causes-of-death. Accessed on 14 Jan 2021.
4. Singapore Cancer Registry 50th Anniversary Monograph 1968 - 2017. Available at: https://www.nrdo.gov.sg/docs/librariesprovider3/defaultdocument-library/thespore-cancerregistry_commerativebook_-1. pdf?sfvrsn=231fce6e_0. Accessed on 14 Jan 2021.

5. Pilleron S, Sarfati D, Janssen-Heijnen M, et al. Global cancer incidence in older adults, 2012 and 2035: A population-based study. Int J Cancer 2019; $144: 49-58$

6. Institute of Medicine (US) Committee on Cancer Control in Low- and Middle-Income Countries. In: Sloan FA, Gelband H (Ed). Cancer control opportunities in low- and middle-income countries. Washington (DC): National Academies Press (US); 2007.

7. Bakoun Z, Hawley JE, Choueiri TK, et al. COVID-19 and Cancer: Current Challenges and Perspectives. Cancer Cell 2020;38:629-46.

8. Upadhaya S, Yu JX, Oliva C, et al. Impact of COVID-19 on oncology clinical trials. Nat Rev Drug Discov 2020;19:376-7.

9. Hanna TP, King WD, Thibodeau S, et al. Mortality due to cancer treatment delay: systematic review and meta-analysis. BMJ 2020;371:m4087.

10. Chiang J, Yang VS, Han S, et al. Minimizing transmission of COVID-19 while delivering optimal cancer care in a National Cancer Centre. J Cancer Policy 2020;25:100241.

11. Cancer versus COVID-19: a Coordinated Disease Disease Outbreak Response System (DORS) to Combat COVID-19 at the National Cancer Centre Singapore. Ann Acad Med Singap 2020:807-9. 